\title{
Volume Rendering
}

National Cancer Institute

\section{Source}

National Cancer Institute. Volume Rendering. NCI Thesaurus. Code C95002.

A 2D projection of a 3D image using one of a variety of algorithms that achieve a display of otherwise hidden voxel values. 